\section{Case Reports in Neurology}

\title{
Notice of Duplicate Publication
}

This is a notice of duplicate publication for the article "A Case of Pediatric Stroke: Osteosarcoma Embolus in the Internal Carotid Artery" [Case Rep Neurol 2021;13:269-275, DOI: 10.1159/000514089].

Two case reports regarding this patient were published inadvertently, by two different groups involved in different aspects of the patient's case, and who were not aware of the other group's planned publication. Please see the article by Shen et al., entitled "Acute Ischemic Stroke in a Pediatric Patient due to Osteosarcoma Embolism" [Pediatr Neurol. 2021 Jul;120:36-37. DOI: 10.1016/j.pediatrneurol.2021.04.009] for a discussion of the diagnosis, etiology and management of this patient. 